\title{
NUMERICAL SOLUTIONS OF THE NONLINEAR POROUS MEDIA EQUATION BASED ON HIGH EXPLORATION PARTICLE SWARM OPTIMIZATION AND MOVING LEAST SQUARES
}

\author{
Mohammad Javad Mahmoodabadi ${ }^{1 *}$, Ali Reza Ghanizadeh² \\ ${ }^{1}$ Department of Mechanical Engineering, Sirjan University of Technology, Sirjan, Iran \\ ${ }^{2}$ Department of Civil Engineering, Sirjan University of Technology, Sirjan, Iran \\ E-mail: mohammadjavadmahmoodabadi@gmail.com \\ *corresponding author
}

\begin{abstract}
In this study, a new numerical method based on the combination of High Exploration Particle Swarm Optimization (HEPSO) and Moving Least Squares (MLS) is introduced to solve nonlinear porous media equations. The MLS scheme is employed to describe an appropriate discretized function, and the penalty method is implemented to convert the constrained problem into an unconstrained one via satisfying the initial conditions. The identified objective function is minimized by the HEPSO to find the approximated nodal values for the nonlinear porous media equation. In order to illustrate the effectiveness of the HEPSO, the optimization trajectories are compared with those of a Standard Particle Swarm Optimization (SPSO) algorithm. Moreover, comparisons are made between the exact solution and the introduced strategy to expose the accuracy, effectiveness and simplicity of the proposed method.
\end{abstract}

Keywords: Moving least square, penalty method, porous media equation, numerical solution, high exploration particle swarm optimization.

\section{Introduction}

Nonlinear partial differential equations as mathematical models frequently appear in many branches of science, engineering and economy. Many models have no closed form solutions and, hence, there is a need to seek approximate solutions by means of numerical methods. To name just a few, İzgi and Çetin (2018) implemented semi-implicit split-step numerical methods for a class of nonlinear stochastic differential equations with non-Lipschitz drift terms. Firoozjaee and Yousefi (2018) utilized using Ritz approximation and proposed a numerical approach for fractional partial differential equations. Fakhar-Izadi and Dehghan (2018) suggested a numerical approach based on the fully spectral collocation method for nonlinear parabolic partial integrodifferential equations. In order to solve hyperbolic partial differential equations, Doha et al. (2019) successfully applied a shifted Jacobi spectral-Galerkin method. Zhou and Zhang (2019) employed one-leg methods for nonlinear stiff fractional differential equations with Caputo derivatives. Esmaeilbeigi et al. (2019) developed a finite difference based numerical scheme to 
analyze time-dependent stochastic partial differential equations using an especial type of the partition of unity collocation method. Li and Wang (2019) combined Galerkin approaches and finite element methods to numerically solve Caputo-type partial differential equations. Yang et al. (2019) presented numerical blow-up analysis of the linearly implicit Euler method for nonlinear parabolic integro-differential equations. Zenget al. (2019) exploited a novel numerical approach based on the discrete least squares collocation method for two-dimensional nonlinear time-dependent partial differential equations.

On the other hand, porous media equations form an important class of dynamical systems which often occurs in nonlinear heat and mass transfer, combustion theory and flows in porous media problems (Polyanin and Zaitsev, 2004). For instance, it describes unsteady heat transfer in a quiescent medium with the heat diffusivity as a power-law function of a temperature (Pamuk, 2005). Consequently, many attempts have been conducted by researchers to investigate the nonlinear porous media equation in the last decade. For instance, Antoniouk and Arnaudon (2014) developed the variational principle to present the solutions of the weighted porous media equation. Jensen et al. (2014) investigated the porous media equations and resistance coefficients for coastal structures. Duque et al. (2015) studied the moving mesh method to analyze the porous media equation with variable exponent. Campos and Soler (2016) investigated the qualitative behavior and traveling waves for flux-saturated porous media equations arising in optimal mass transportation. Grillo and Muratori (2016) examined smoothing effects for the porous media equation on Cartan-Hadamard manifolds. Röckner et al. (2018) inspected stochastic porous media equation on general measure spaces with increasing Lipschitz nonlinearities. Dareiotis et al. (2019) reconnoitered entropy solutions for stochastic porous media equations. Duan et al. (2019) probed an energetic variational approach to represent numerical solutions for the porous media equation.

In pursuance to this, we propose modifications in the existing methods to solve porous media equations based on the new heuristic method introduced by Mahmoodabadi et al. (2014 and 2018). To this end, the MLS scheme is employed to describe an appropriate discretized function, and the penalty method is implemented to convert the constrained problem into an unconstrained one via satisfying the initial conditions. The identified objective function is minimized by the HEPSO to find the approximated nodal values for the nonlinear porous media equation and results are compared with SPSO. From the obtained results, it is observed that the proposed new method is consistent, stable and accurate to solve the nonlinear porous media equation.

The rest of this paper is structured as follows. Section 2 states the governing nonlinear porous media differential equation. Section 3 briefly introduces the SPSO and HEPSO algorithms. The MLS scheme and discretization of the porous media equation are mentioned in Sections 4 and 5, respectively. The simulation results and the comparisons are discussed in Section 6. Section 7 concludes the results of this study.

\section{Porous media differential equation}

Consider the nonlinear porous media differential equation as follows (Pamuk, 2005).

$$
\frac{\partial \psi}{\partial \tau}=\frac{\partial}{\partial \zeta}\left(\psi^{r} \frac{\partial \psi}{\partial \zeta}\right)
$$

where, $r$ is a rational number, $\zeta$ represents position coordinate, $\tau$ denotes the time, and $\psi$ signifies desired variable of the equation. Let us respect $r=1$ in Eq. (1); therefore, the equation is rewritten as follows.

$$
\frac{\partial \psi}{\partial \tau}=\frac{\partial}{\partial \zeta}\left(\psi \frac{\partial \psi}{\partial \zeta}\right),
$$


with the initial condition of

$$
\psi(\zeta, 0)=\psi_{\zeta, 0}
$$

and boundary conditions of

$$
\psi(0, \tau)=\psi_{0, \tau} \text { and } \psi(1, \tau)=\psi_{1, \tau}
$$

\section{High exploration particle swarm optimization}

The general form of the Standard PSO (SPSO) equations could be written as follows (Sibalija, 2019):

$$
\begin{gathered}
\vec{\phi}_{i}(t+1)=w \vec{\phi}_{i}(t)+\alpha \vec{r}_{1}\left(\vec{\psi}_{\text {pbest }_{i}}(t)-\vec{\psi}_{i}(t)\right)+\beta \vec{r}_{2}\left(\vec{\psi}_{\text {gbest }}(t)-\vec{\psi}_{i}(t)\right) \\
\vec{\psi}_{i}(t+1)=\vec{\psi}_{i}(t)+\vec{\phi}_{i}(t+1)
\end{gathered}
$$

where, $\vec{\psi}_{i}(t)$ and $\vec{\phi}_{i}(t)$ describe the position and velocity of the particle $i$ at certain iteration $t$, respectively. Coefficients $\alpha$ and $\beta$ denote the tendency of the particle to the personal and social successes, respectively $\overrightarrow{r_{1}}$ and $\overrightarrow{r_{2}}$ are random vectors in interval [0,1], and $w$ illustrates the effect of the particle velocity at the previous iteration on the next one. Furthermore, $\vec{\psi}_{\text {pbest }_{i}}(t)$ and $\vec{\psi}_{\text {gbest }}(t)$ are personal and global best positions of the particle $i$ and the swarm at iteration $t$, respectively.

In the HEPSO algorithm, two operators are added to the PSO equations. The first one is based on the multi-crossover mechanism of the genetic algorithm and the other one is obtained via artificial bee colony.

Genetic algorithm operation: According to multiple-crossover idea, a new formulation for the velocity of a particle is proposed as follows:

$$
\vec{\phi}_{i}(t+1)=\vec{r}\left(\frac{\beta}{2} \vec{\psi}_{\text {gbest }}(t)-\vec{\psi}_{\text {pbest }_{i}}(t)-\vec{\psi}_{i}(t)\right),
$$

where, $\vec{r}$ is a vector of random values between $[0,1]$.

Artificial bee colony operation: The main operator of the artificial bee colony algorithm is utilized to determine the new position of particle $i$ in dimension $d$ as the following equation.

$$
\psi_{i}^{d}(t+1)=\psi_{i}^{d}(t)+(2 r-1)\left(\psi_{i}^{d}(t)-\psi_{j}^{d}(t)\right),
$$

where, $r$ is a random value between [0,1]. $j$ is an integer random number between 1 and the maximum number of particles.

\section{Moving least square}

The moving least square approximation is formulized in this section. At first, the shape function $\chi\left(\mathrm{x}_{i}\right)$ for a selected point $\mathrm{x}_{i}=\left[\tau_{i}, \zeta_{i}\right]^{T} \in \Psi$ is defined by:

$$
\chi\left(\mathrm{x}_{i}\right)=\omega^{T}\left(\mathrm{x}_{i}\right) \Gamma^{-1}(\mathrm{x}) \mathrm{E}(\mathrm{x}) \text { for } \mathrm{x} \in \Psi_{d},
$$

where, $\omega^{T}\left(\mathrm{x}_{i}\right)=\left[1, \mathrm{x}_{i}^{1}, \mathrm{x}_{i}^{2}, \mathrm{x}_{i}^{3}, \ldots, \mathrm{x}_{i}^{m}\right]$ is the monomial basis vector of order $m$; and matrices $\Gamma(\mathrm{x})$ and $\mathrm{E}(\mathrm{x})$ are calculated by the following relations.

$$
\Gamma(\mathrm{x})=\Omega^{T} \Lambda \Omega,
$$




$$
\mathrm{E}(\mathrm{x})=\Omega^{T} \Lambda,
$$

where, $\Omega=\left[\omega\left(\mathrm{x}_{1}\right), \omega\left(\mathrm{x}_{2}\right), \ldots, \omega\left(\mathrm{x}_{n}\right)\right]^{T}$ is the monomial basis matrix; $n$ refers to the number of nodes in the supporting domain $\Psi_{\mathrm{x}}$ of the global domain $\Psi$; and $\Lambda$ represents the diagonal weight function matrix whose $\mathrm{j}$ th diagonal member is calculated by the following equation.

$$
\lambda_{j}\left(\mathrm{x}_{i}\right)=\left\{\begin{array}{cc}
1-6\left(\frac{\bar{\rho}_{i j}}{\rho}\right)^{2}+8\left(\frac{\bar{\rho}_{i j}}{\rho}\right)^{3}-3\left(\frac{\bar{\rho}_{i j}}{\rho}\right)^{4} & 0 \leq \bar{\rho}_{i j} \leq \rho \\
0 & \bar{\rho}_{i j} \geq \rho
\end{array},\right.
$$

where, $j=1,2, \ldots, n ; \bar{\rho}_{i j}$ is the distance between points $\mathrm{x}_{i}$ and $\mathrm{x}_{j}$; and $\rho$ represents the size of the supporting domain. After calculation of the shape function $\chi\left(x_{i}\right)$, the unknown value $\psi^{h}\left(x_{i}\right)$ is approximated from the nodal values $\hat{\psi}(\mathrm{x})$ as bellow (Mahmoodabadi et al., 2018)

$$
\psi^{h}\left(\mathrm{x}_{i}\right)=\chi\left(\mathrm{x}_{i}\right) \hat{\psi}(\mathrm{x}) .
$$

\section{Discretization of the porous media equation}

In this section, the objective function of the problem is identified for minimization by the detailed HEPSO algorithm. Suppose that $\mathrm{x}_{i}=\left[\tau_{i}, \zeta_{i}\right]^{T}$ denotes $i$ th nodal position on the problem domain, and $\hat{\psi}\left(\mathrm{x}_{i}\right)$ indicates the approximation function value at $\mathrm{x}_{i}$. Using Eq. (13), Eq. (2) could be rewritten for $i$ th node as follows.

$$
\frac{d \chi\left(\mathrm{x}_{i}\right)}{d \tau} \hat{\psi}\left(\mathrm{x}_{i}\right)-\frac{d \chi\left(\mathrm{x}_{i}\right)}{d \zeta}\left[\hat{\psi}\left(\mathrm{x}_{i}\right)\left(\frac{d \chi\left(\mathrm{x}_{i}\right)}{d \zeta} \hat{\psi}\left(\mathrm{x}_{i}\right)\right)\right]=0
$$

The summation of absolute values of these equations for all nodal points generates the first part of the discretized objective function.

$$
\sum_{i=1}^{M}\left|\frac{d \chi\left(\mathrm{x}_{i}\right)}{d \tau} \hat{\psi}\left(\mathrm{x}_{i}\right)-\frac{d \chi\left(\mathrm{x}_{i}\right)}{d \zeta}\left[\hat{\psi}\left(\mathrm{x}_{i}\right)\left(\frac{d \chi\left(\mathrm{x}_{i}\right)}{d \zeta} \hat{\psi}\left(\mathrm{x}_{i}\right)\right)\right]\right|=0
$$

where, $M$ is the total number of nodes.

If the penalty method is utilized to impose the initial and boundary conditions, then:

$$
\sum_{i=1}^{M}\left|\frac{d \chi\left(\mathrm{x}_{i}\right)}{d \tau} \hat{\psi}\left(\mathrm{x}_{i}\right)-\frac{d \chi\left(\mathrm{x}_{i}\right)}{d \zeta}\left[\hat{\psi}\left(\mathrm{x}_{i}\right)\left(\frac{d \chi\left(\mathrm{x}_{i}\right)}{d \zeta} \hat{\psi}\left(\mathrm{x}_{i}\right)\right)\right]\right|+\Gamma_{1}\left|\psi(\zeta, 0)-\psi_{\zeta, 0}\right|+\Gamma_{2} \mid \psi(0, \tau)-
$$
$\psi_{0, \tau}\left|+\Gamma_{3}\right| \psi(1, \tau)-\psi_{1, \tau} \mid=0$

where, $\Gamma_{i}(i=1,2,3) \gg 1$ are the penalty parameters.

\section{Numerical results and comparisons}

In this section, the introduced strategy based on the HEPSO and MLS is applied to analyze the porous media nonlinear differential equation illustrated by Eq. (2). If $M$ is the total number of nodes, the population size of optimization algorithm is considered as $10 \times M$; its maximum number of iterations is set as $500 \times M$, while the penalty parameters are set at $\Gamma_{i}=100(i=$ $1,2,3)$. The characteristics of the SPSO and HEPSO algorithms are presented in Table 1 . The global domain is considered as $\tau \in[0,1]$ and $\zeta \in[0,1]$ with the supporting domain size $\rho=$ 
1.001 for different distances between nodal points in $\tau$ direction $\left(h_{\tau}\right)$ and $\zeta$ direction $\left(h_{\zeta}\right)$. The problem is solved for several different initial and boundary conditions illustrated in the following examples.

\subsection{Illustrative Example 1}

Consider the problem of the porous media with governing Eq. (2) and the following initial and boundary conditions:

$$
\psi(\zeta, 0)=\zeta, \psi(0, \tau)=\tau, \psi(1, \tau)=1+\tau
$$

The exact solutions for this problem is as follows:

$$
\psi(\zeta, \tau)=\zeta+\tau
$$

\begin{tabular}{|c|c|}
\hline Algorithm & Parameters \\
\hline SPSO & $\alpha=3 ; \beta=3 ; w=1$ \\
\hline \multirow{2}{*}{ HEPSO } & $\alpha_{1 i}=2.5 ; \alpha_{1 f}=0.5 ; \beta_{2 i}=0.5 ; \beta_{2 i}=2.5 ; w=$ adaptive; $P_{C}=$ \\
& $0.95 ; P_{B}=0.02$ \\
\hline
\end{tabular}

Table 1. Parameter configurations of the optimization algorithms.

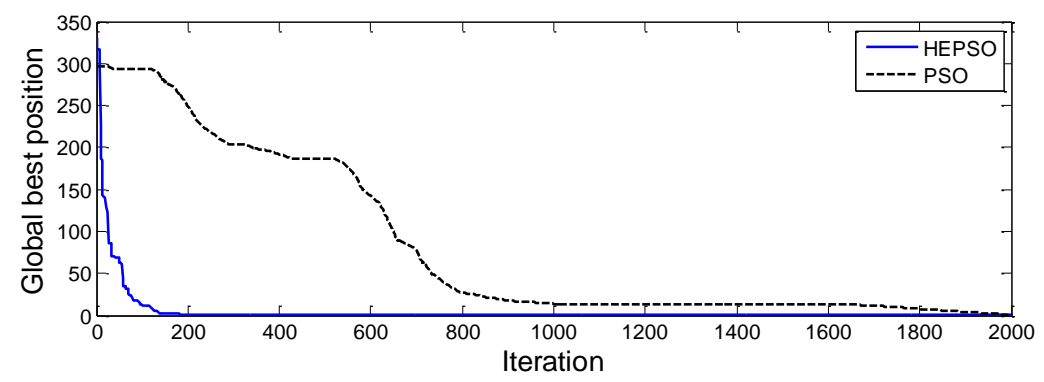

Fig. 1. Convergence trajectory of SPSO and HEPSO for $h_{\tau}=0.5$ and $h_{\zeta}=0.5$ (Example 1 ).

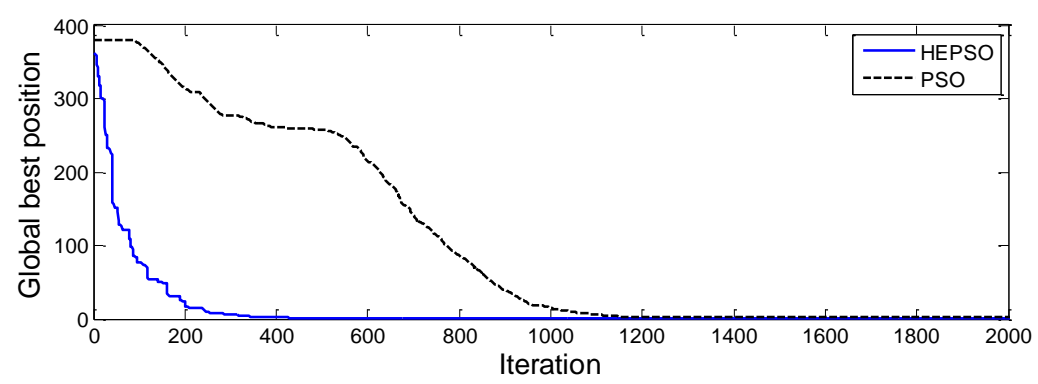

Fig. 2. Convergence trajectory of SPSO and HEPSO for $h_{\tau}=0.5$ and $h_{\zeta}=0.25$ (Example 1). 


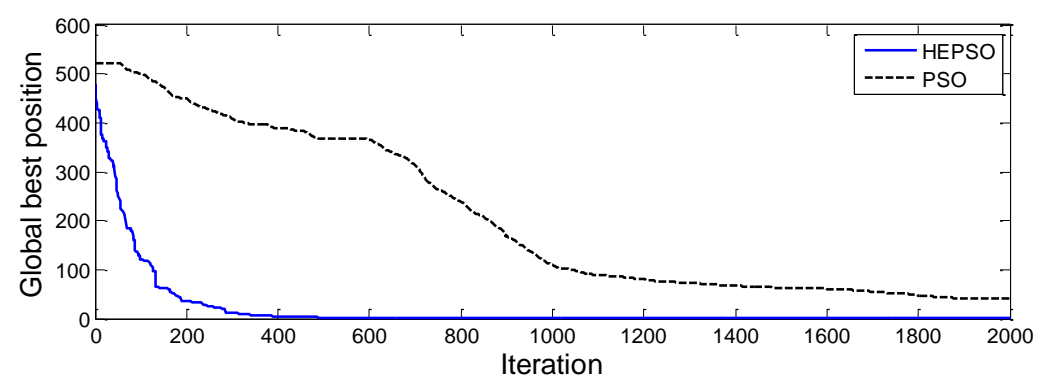

Fig. 3. Convergence trajectory of SPSO and HEPSO for $h_{\tau}=0.5$ and $h_{\zeta}=0.2$ (Example 1 ).

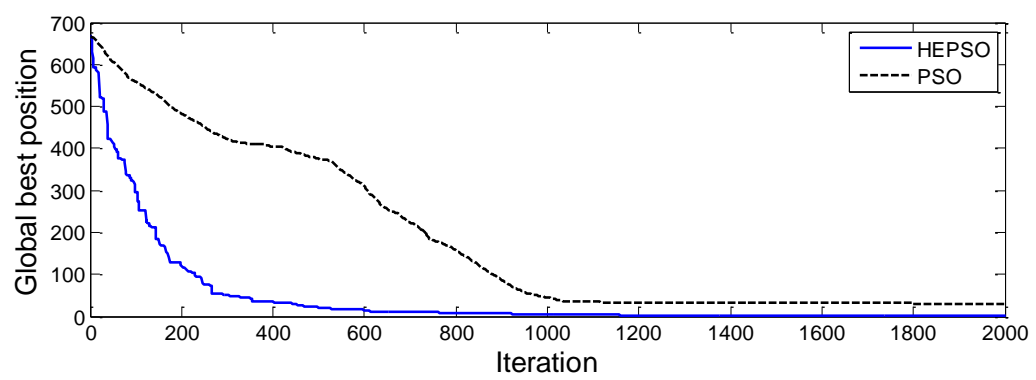

Fig. 4. Convergence trajectory of SPSO and HEPSO for $h_{\tau}=0.5$ and $h_{\zeta}=0.125$ (Example 1 ).

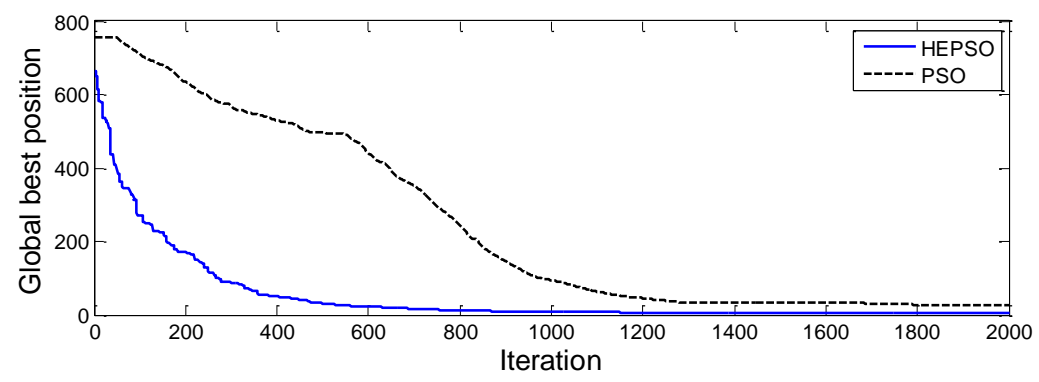

Fig. 5. Convergence trajectory of SPSO and HEPSO for $h_{\tau}=0.5$ and $h_{\zeta}=0.1$ (Example 1 ).

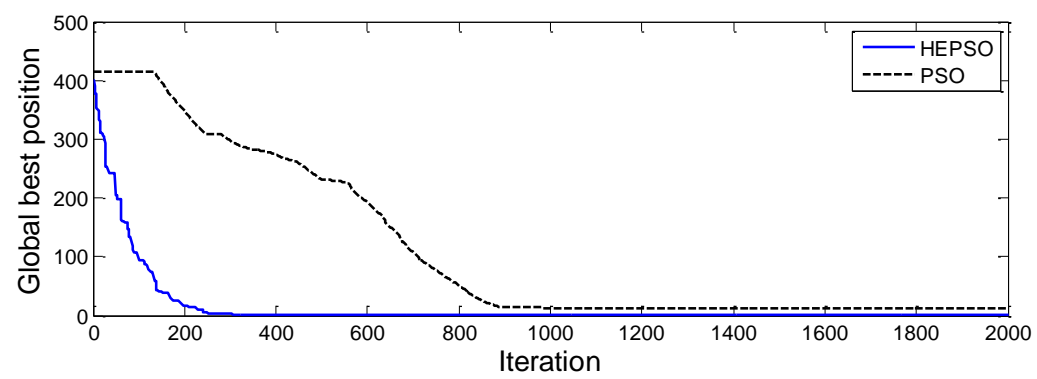

Fig. 6. Convergence trajectory of SPSO and HEPSO for $h_{\tau}=0.25$ and $h_{\zeta}=0.5$ (Example 1). 


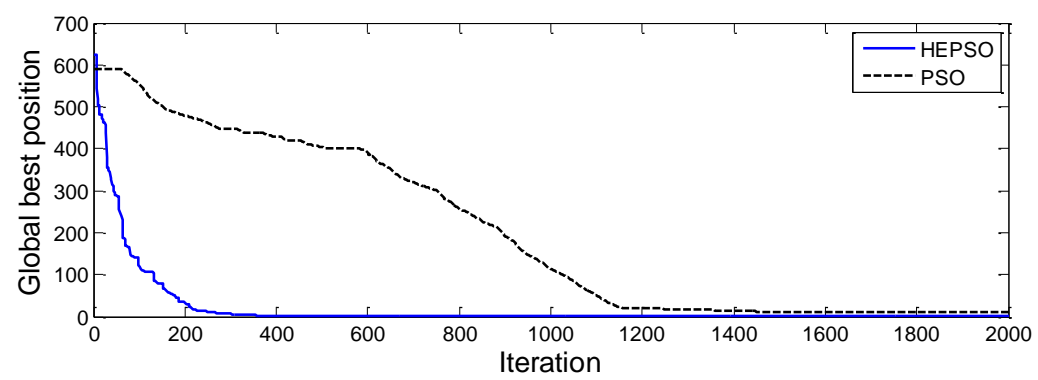

Fig. 7. Convergence trajectory of SPSO and HEPSO for $h_{\tau}=0.2$ and $h_{\zeta}=0.5$ (Example 1).

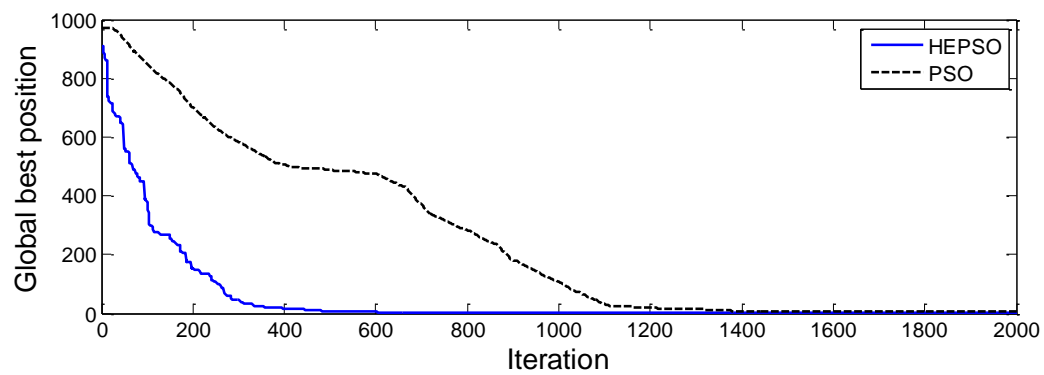

Fig. 8. Convergence trajectory of SPSO and HEPSO for $h_{\tau}=0.125$ and $h_{\zeta}=0.5$ (Example 1).

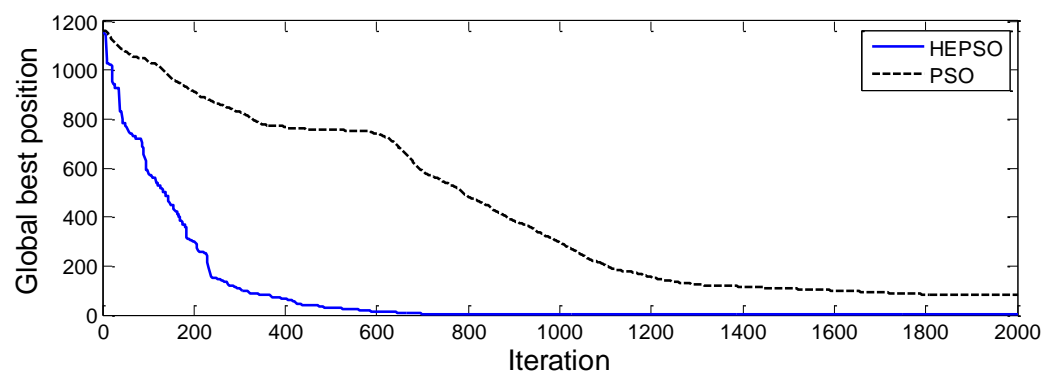

Fig. 9. Convergence trajectory of SPSO and HEPSO for $h_{\tau}=0.1$ and $h_{\zeta}=0.5$ (Example 1$)$. 


\begin{tabular}{|c|c|c|c|c|c|}
\hline$h_{\tau}$ & $h_{\zeta}$ & $\begin{array}{c}\text { Objective function of } \\
\text { HEPSO }\end{array}$ & $\begin{array}{c}\text { Objective function } \\
\text { of SPSO }\end{array}$ & $\begin{array}{c}\text { Error of } \\
\text { HEPSO }\end{array}$ & $\begin{array}{c}\text { Error of } \\
\text { SPSO }\end{array}$ \\
\hline \multirow{3}{*}{0.5} & 0.1 & 0.0060136 & 9.1232 & $9.4037 \mathrm{e}-05$ & 0.07413 \\
\cline { 2 - 6 } & 0.125 & 0.0032618 & 6.2153 & $5.7964 \mathrm{e}-05$ & 0.08864 \\
\cline { 2 - 6 } & 0.2 & 0.00036714 & 2.7687 & $8.8421 \mathrm{e}-06$ & 0.040681 \\
\cline { 2 - 6 } & 0.25 & 0.00032032 & 2.1042 & $7.0401 \mathrm{e}-06$ & 0.014804 \\
\cline { 2 - 6 } & 0.5 & 0.005225 & 0.19557 & $3.4672 \mathrm{e}-05$ & 0.00055015 \\
\hline \multirow{2}{*}{0.1} & \multirow{3}{*}{0.5} & 0.013239 & 1.4875 & $4.1813 \mathrm{e}-05$ & 0.0012832 \\
\cline { 3 - 6 } & & 0.011228 & 0.65529 & $4.3519 \mathrm{e}-05$ & 0.00056184 \\
\hline 0.2 & & 0.008176 & 0.2854 & $4.2103 \mathrm{e}-05$ & 0.00044996 \\
\hline 0.25 & & 0.007133 & 0.23361 & $3.9744 \mathrm{e}-05$ & 0.00036228 \\
\hline
\end{tabular}

Table 2. Objective functions and errors for different distances between nodal points in directions $\tau$ and $\zeta$ (Example 1).

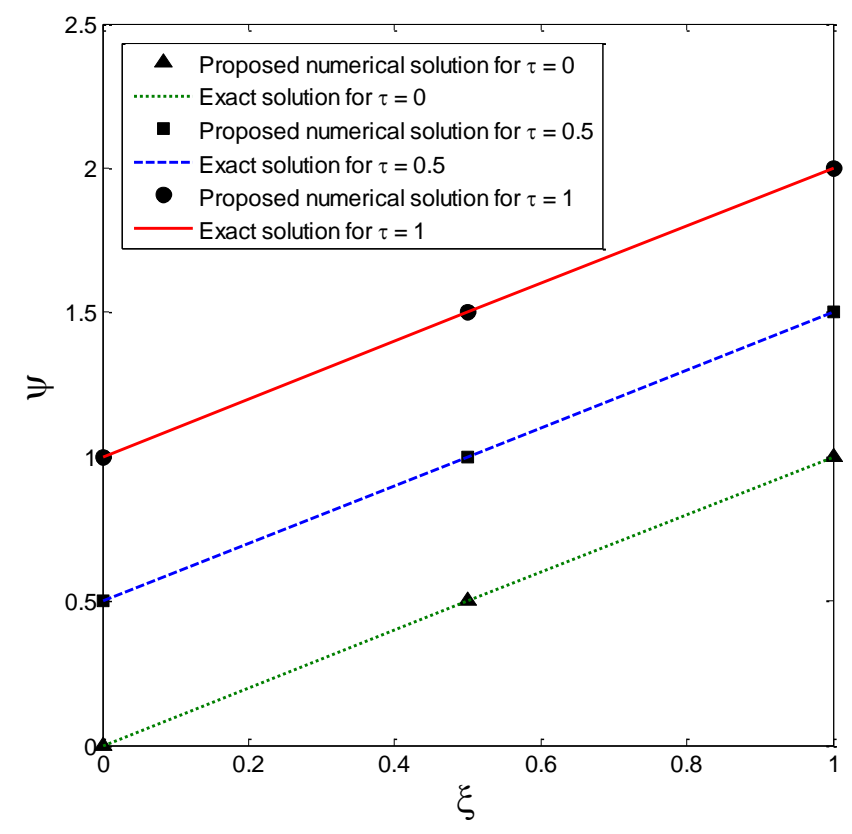

Fig. 10. Comparison of results obtained via the proposed method and exact solution for $h_{\tau}=$ 0.5 and $h_{\zeta}=0.5$ (Example 1$)$. 


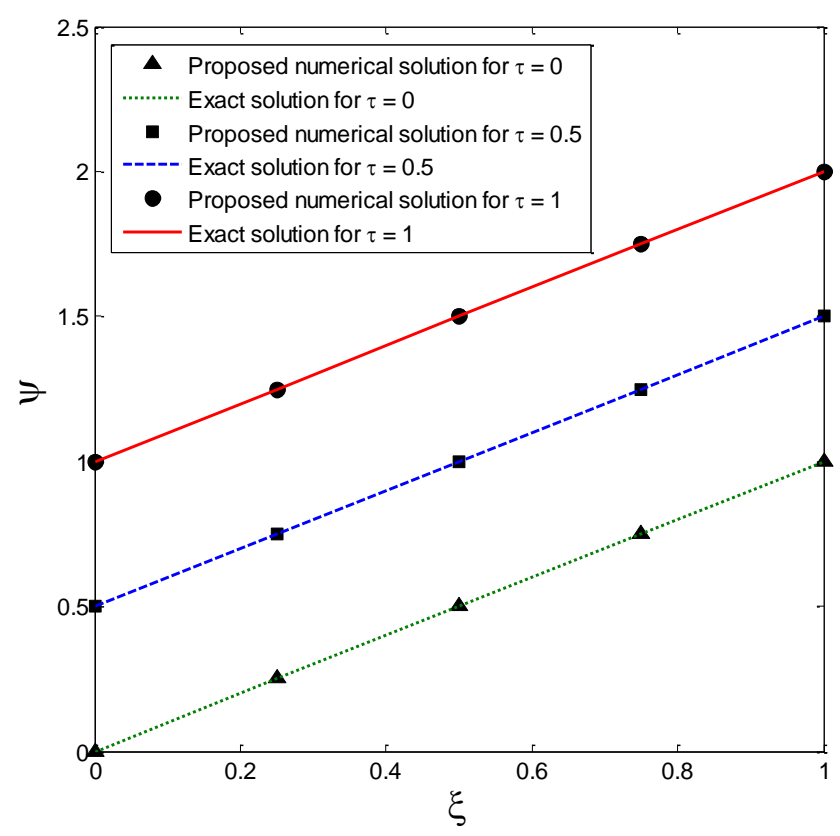

Fig. 11. Comparison of results obtained via the proposed method and exact solution for $h_{\tau}=$ 0.5 and $h_{\zeta}=0.25$ (Example 1).

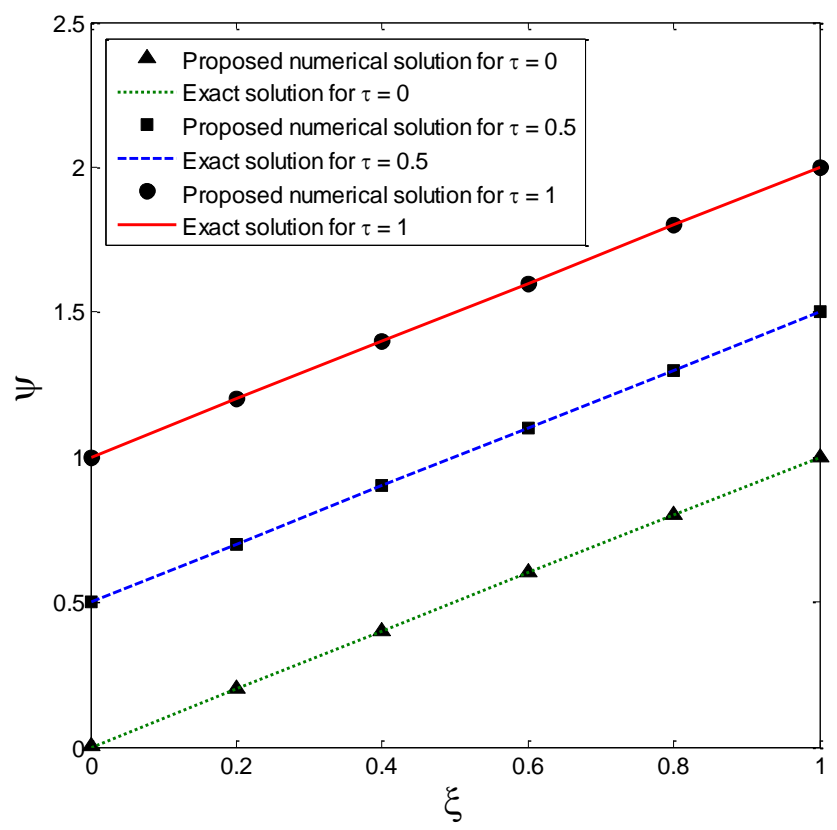

Fig. 12. Comparison of results obtained via the proposed method and exact solution for $h_{\tau}=$ 0.5 and $h_{\zeta}=0.2$ (Example 1). 


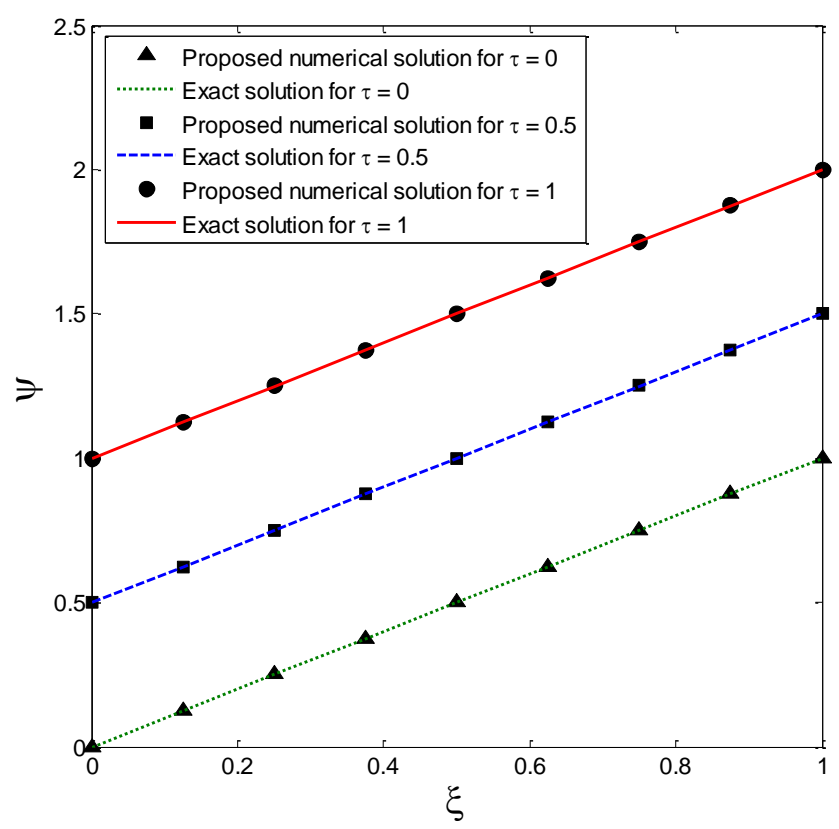

Fig. 13. Comparison of results obtained via the proposed method and exact solution for $h_{\tau}=$ 0.5 and $h_{\zeta}=0.125$ (Example 1).

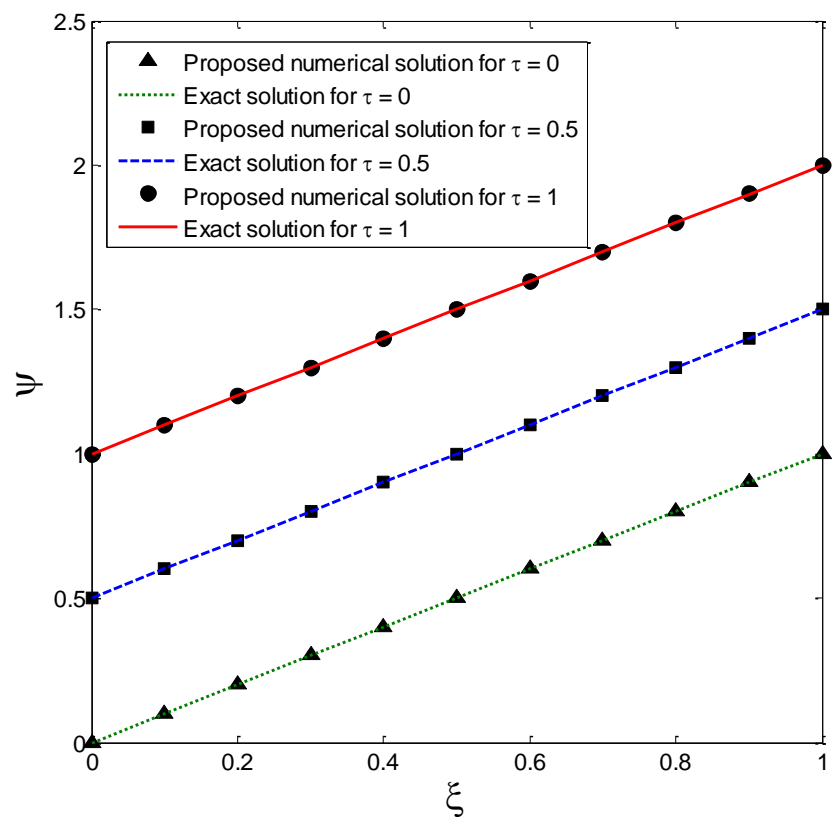

Fig. 14. Comparison of results obtained via the proposed method and exact solution for $h_{\tau}=$ 0.5 and $h_{\zeta}=0.1$ (Example 1). 


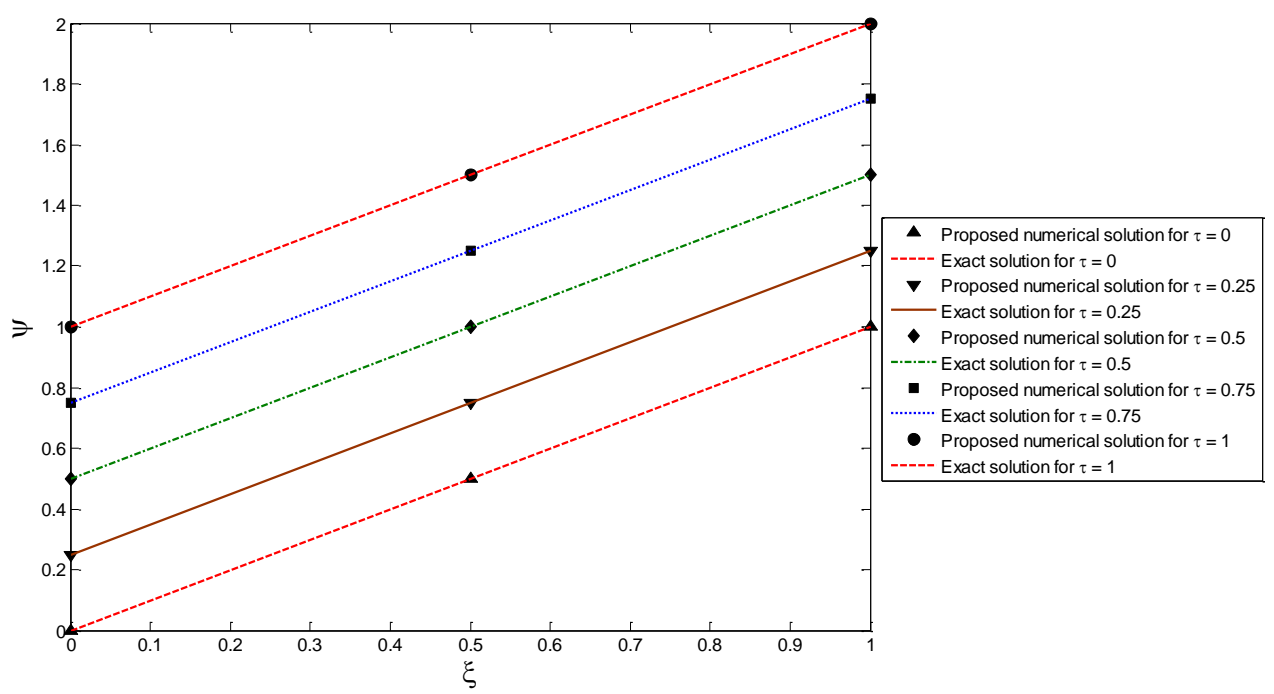

Fig. 15. Comparison of results obtained via the proposed method and exact solution for $h_{\tau}=$ 0.25 and $h_{\zeta}=0.5$ (Example 1).

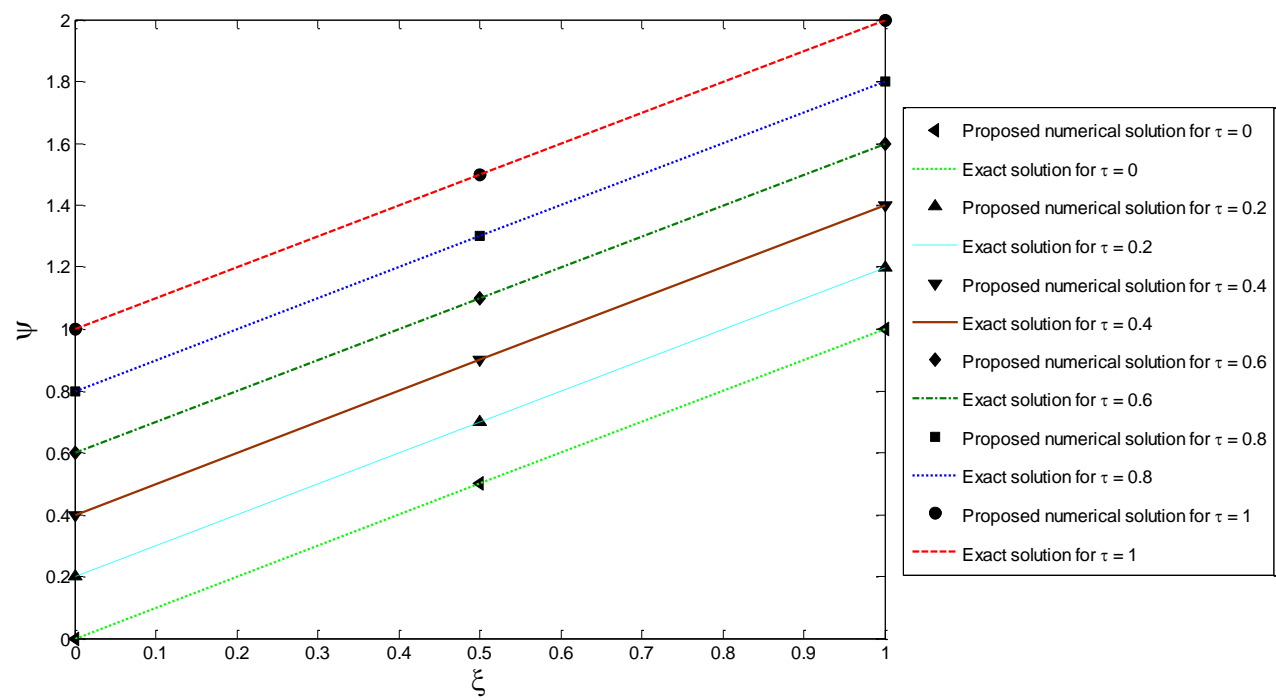

Fig. 16. Comparison of results obtained via the proposed method and exact solution for $h_{\tau}=$ 0.2 and $h_{\zeta}=0.5$ (Example 1). 


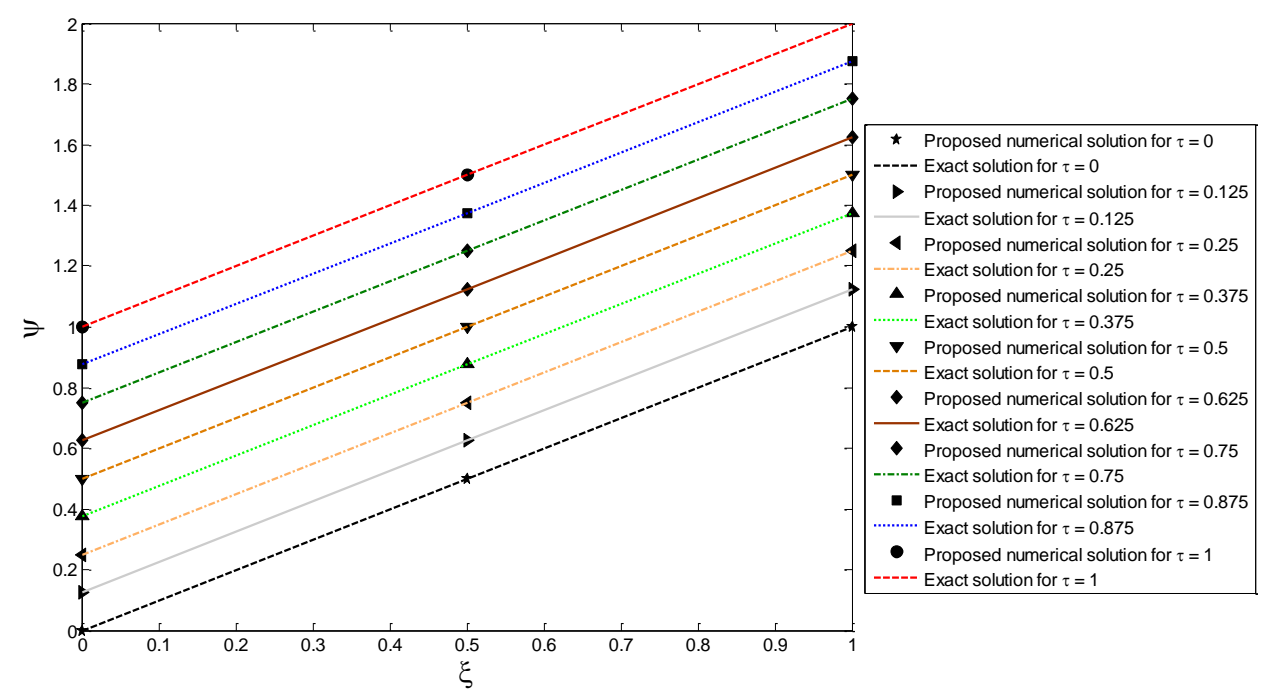

Fig. 17. Comparison of results obtained via the proposed method and exact solution for $h_{\tau}=$ 0.125 and $h_{\zeta}=0.5$ (Example 1).

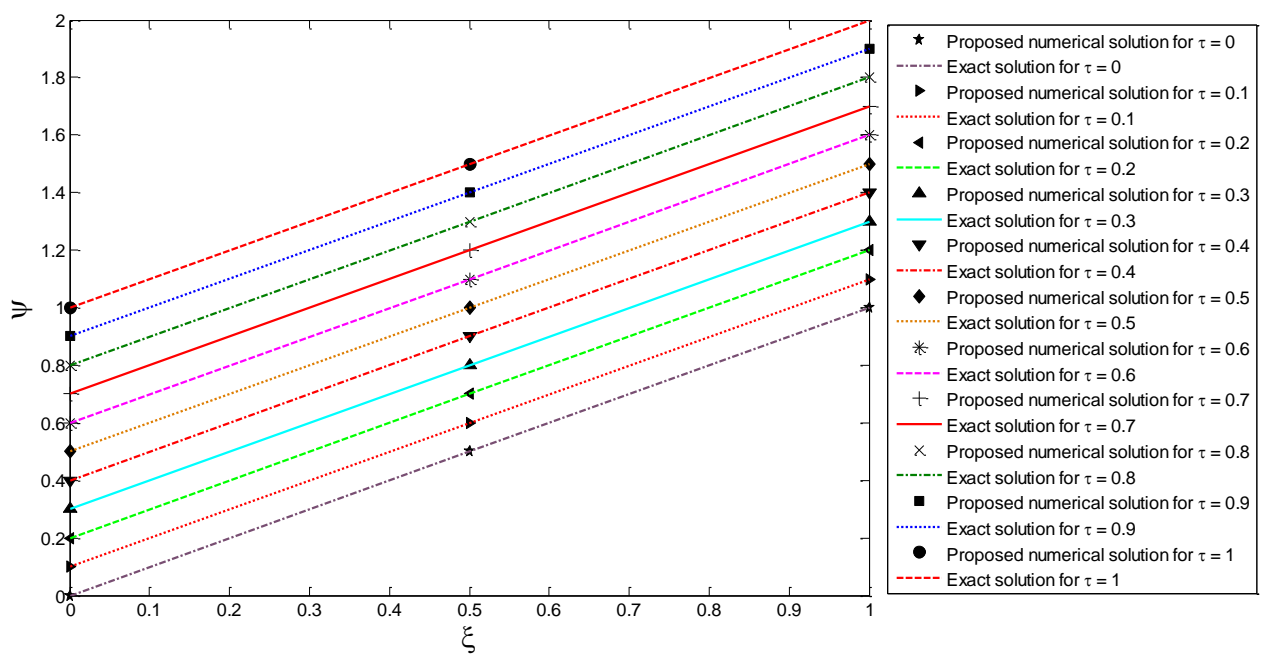

Fig. 18. Comparison of results obtained via the proposed method and exact solution for $h_{\tau}=$ 0.1 and $h_{\zeta}=0.5$ (Example 1).

The convergence trajectories of the SPSO and HEPSO are shown in Figs. 1 through 9 for different distances between nodal points (for more visibility, only the first 2000 iterations are exhibited). Further, the objective functions obtained by SPSO and HEPSO for the global best particle as well as the related errors are illustrated in Table 2. This table clearly depicts that the HEPSO approach is able to find better results in comparison with the SPSO method. Additionally, the numerical solutions found via the MLS based PSO scenario are compared with those of the 
exact solution in Figs. 10 to 18 for different nodal distributions. These comparisons obviously portray the high accuracy, great stability and excellent convergence of the proposed strategy to solve the porous media nonlinear differential equation.

\subsection{Illustrative Example 2}

The governing porous media equation was considered as Eq. (2) and the initial and boundary conditions are regarded as follows.

$$
\psi(\zeta, 0)=-\frac{\zeta^{2}}{6}, \psi(0, \tau)=0, \psi(1, \tau)=-\frac{1}{6(1+\tau)}
$$

The exact solution related to the above equation could be given as Eq. (20).

$$
\psi(\zeta, \tau)=-\frac{\zeta^{2}}{6(1+\tau)}
$$

The convergence trajectories obtained by the regarded optimization algorithms (SPSO and HEPSO) are indicated in Figs. 19 through 27 for different number of the nodal points (for more visibility, only the first 5000 iterations are disclosed). Additionally, the final value of objective function of the global best particle and the related errors are represented in Table 3. This table clearly depicts that the HEPSO approach is able to find better results in comparison with the SPSO method. As well, the numerical solutions originated via the MLS based PSO method and the analytical approach are demonstrated in Figs. 28 to 36 for the various nodal distributions.

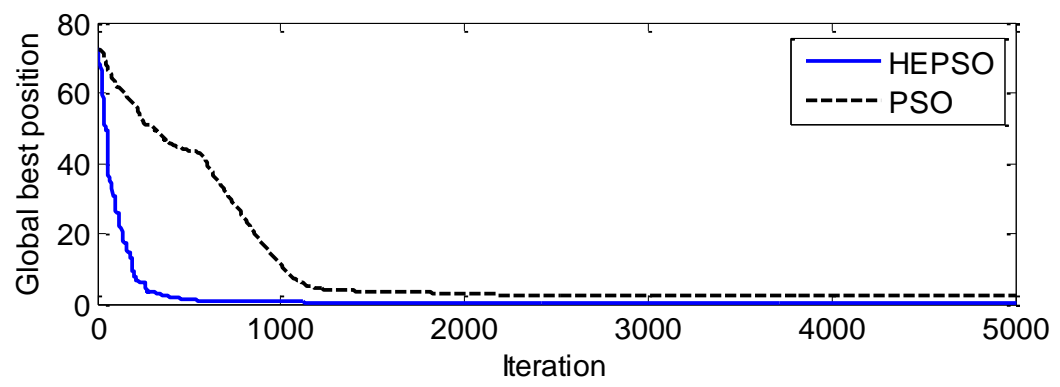

Fig. 19. Convergence trajectory of SPSO and HEPSO for $h_{\tau}=0.25$ and $h_{\zeta}=0.25$ (Example $2)$.

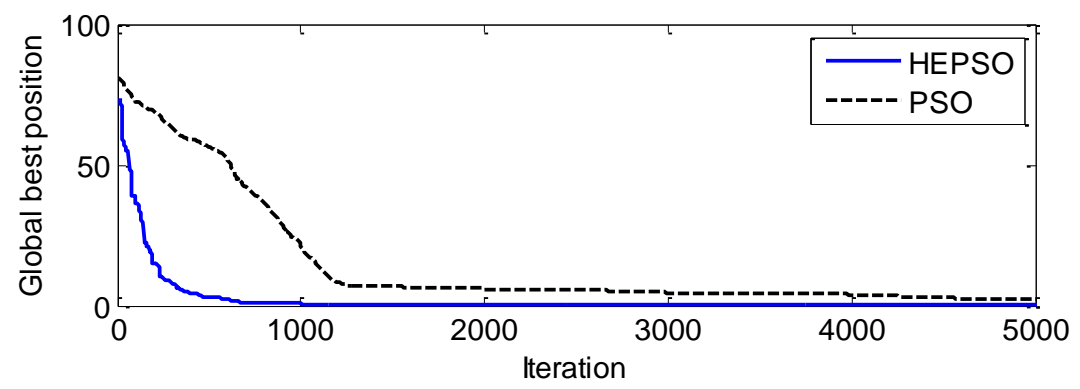

Fig. 20. Convergence trajectory of SPSO and HEPSO for $h_{\tau}=0.25$ and $h_{\zeta}=0.2$ (Example 2). 


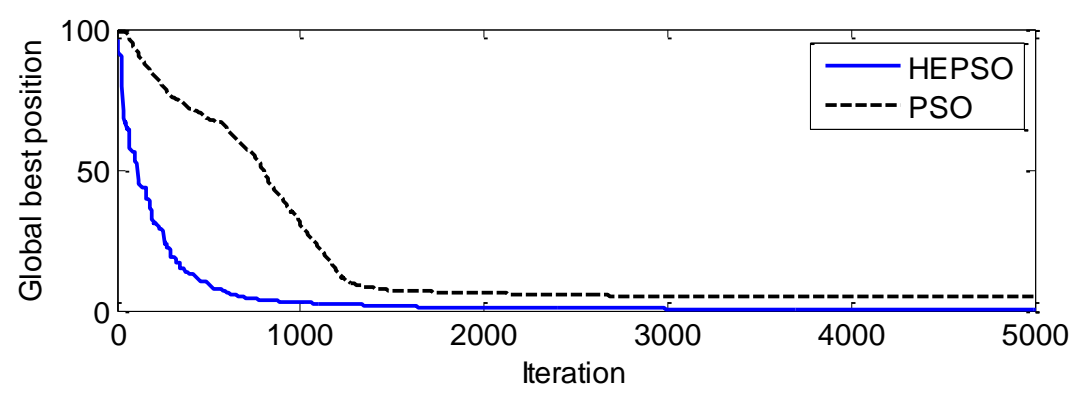

Fig. 21. Convergence trajectory of SPSO and HEPSO for $h_{\tau}=0.25$ and $h_{\zeta}=0.125$ (Example 2).

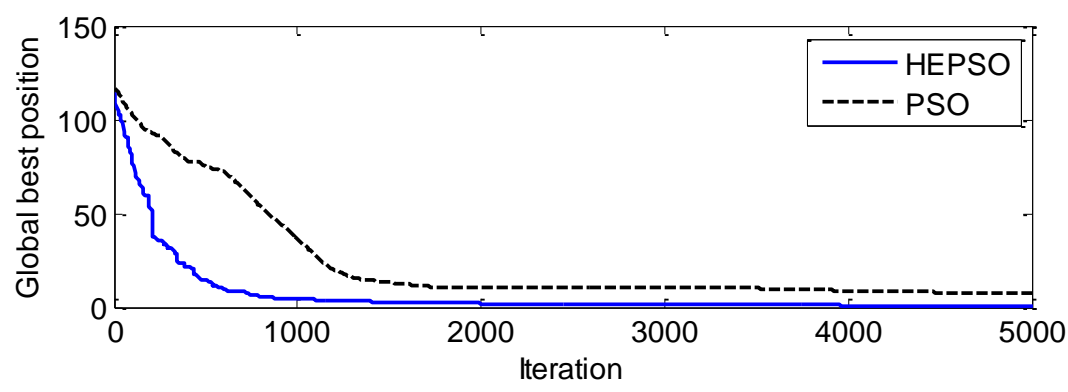

Fig. 22. Convergence trajectory of SPSO and HEPSO for $h_{\tau}=0.25$ and $h_{\zeta}=0.1$ (Example 2).

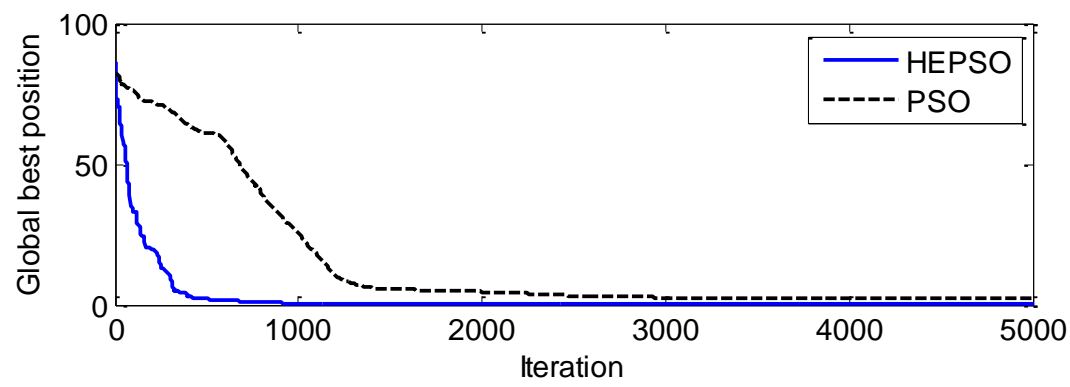

Fig. 23. Convergence trajectory of SPSO and HEPSO for $h_{\tau}=0.2$ and $h_{\zeta}=0.25$ (Example 2). 


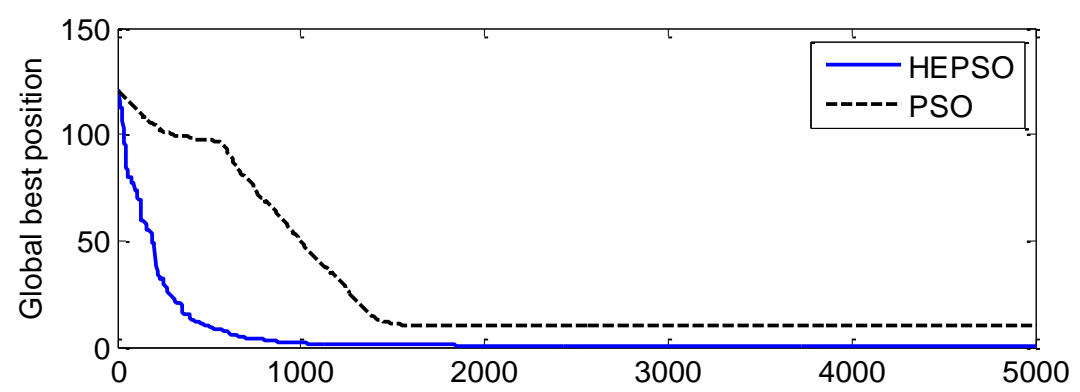

Fig. 24. Convergence trajectory of SPSO and HEPSO for $h_{\tau}=0.125$ and $h_{\zeta}=0.25$ (Example $2)$.

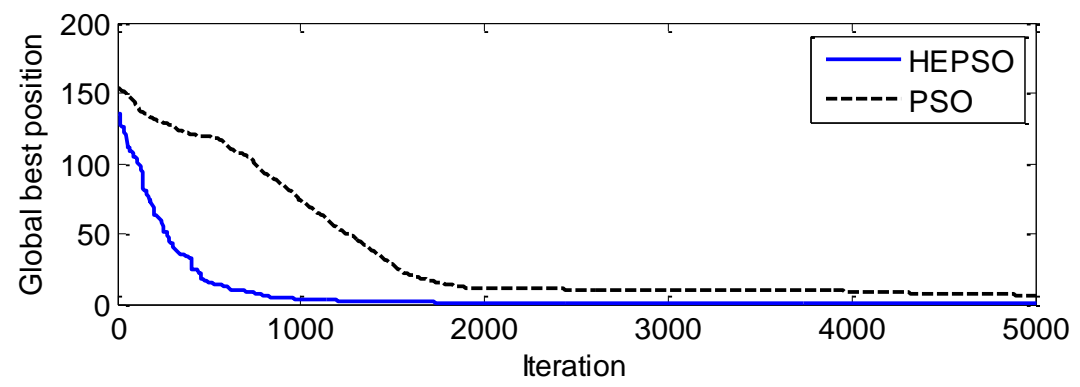

Fig. 25. Convergence trajectory of SPSO and HEPSO for $h_{\tau}=0.1$ and $h_{\zeta}=0.25$ (Example 2).

\begin{tabular}{|c|c|c|c|c|c|}
\hline$h_{\tau}$ & $h_{\zeta}$ & $\begin{array}{c}\text { Objective function } \\
\text { of HEPSO }\end{array}$ & $\begin{array}{c}\text { Objective function } \\
\text { of SPSO }\end{array}$ & $\begin{array}{c}\text { Error of } \\
\text { HEPSO }\end{array}$ & $\begin{array}{c}\text { Error of } \\
\text { SPSO }\end{array}$ \\
\hline \multirow{3}{*}{0.25} & 0.1 & 0.056981 & 3.7632 & 0.00082616 & 0.03264 \\
\cline { 2 - 6 } & 0.125 & 0.015525 & 3.2015 & 0.00014218 & 0.030369 \\
\cline { 2 - 6 } & 0.2 & 0.010368 & 2.4376 & $7.6648 \mathrm{e}-05$ & 0.024178 \\
\cline { 2 - 6 } & 0.25 & 0.0095065 & 2.2788 & $7.9057 \mathrm{e}-05$ & 0.026548 \\
\hline 0.1 & \multirow{3}{*}{0.25} & 0.089099 & 4.6552 & 0.0042164 & 0.03105 \\
\cline { 3 - 6 } & & 0.018598 & 3.574 & 0.00082393 & 0.029527 \\
\cline { 3 - 6 } 0.125 & & 0.008846 & 1.6514 & $8.4606 \mathrm{e}-05$ & 0.023025 \\
\hline 0.2 & & & & & \\
\hline
\end{tabular}

Table 3. Objective functions and errors for different distances between nodal points in directions $\tau$ and $\zeta$ (Example 2). 


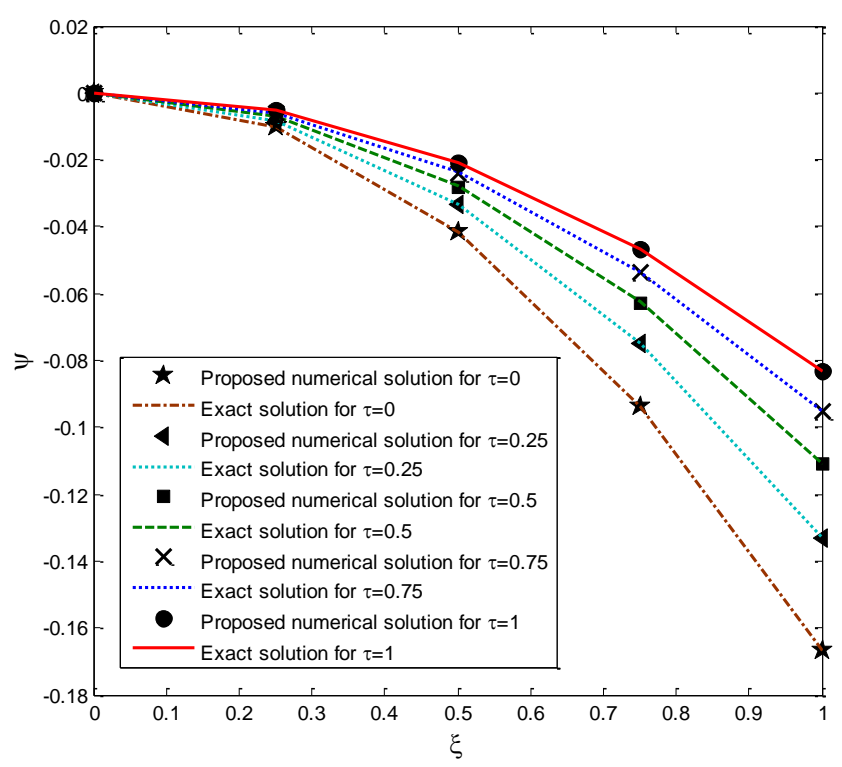

Fig. 26. Comparison of results obtained via the proposed method and exact solution for $h_{\tau}=$ 0.25 and $h_{\zeta}=0.25$ (Example 2).

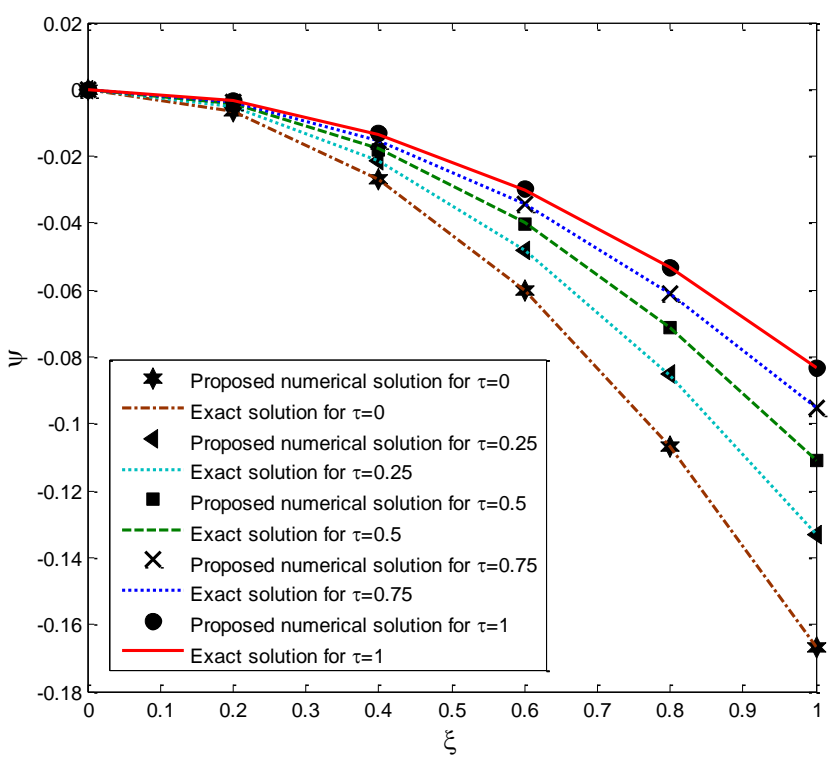

Fig. 27. Comparison of results obtained via the proposed method and exact solution for $h_{\tau}=$ 0.25 and $h_{\zeta}=0.2$ (Example 2). 


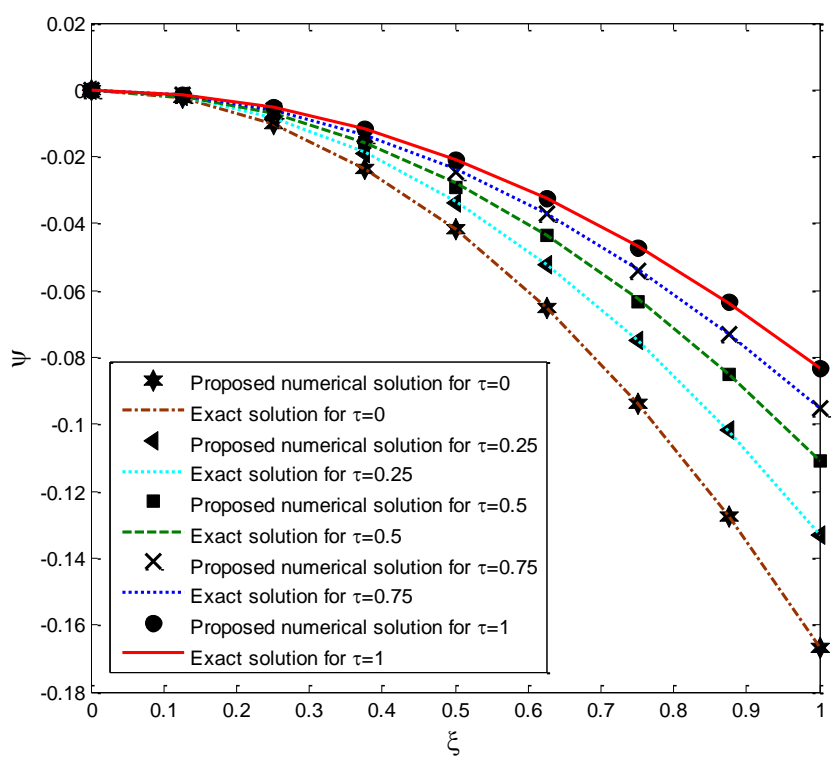

Fig. 28. Comparison of results obtained via the proposed method and exact solution for $h_{\tau}=$ 0.25 and $h_{\zeta}=0.125$ (Example 2).

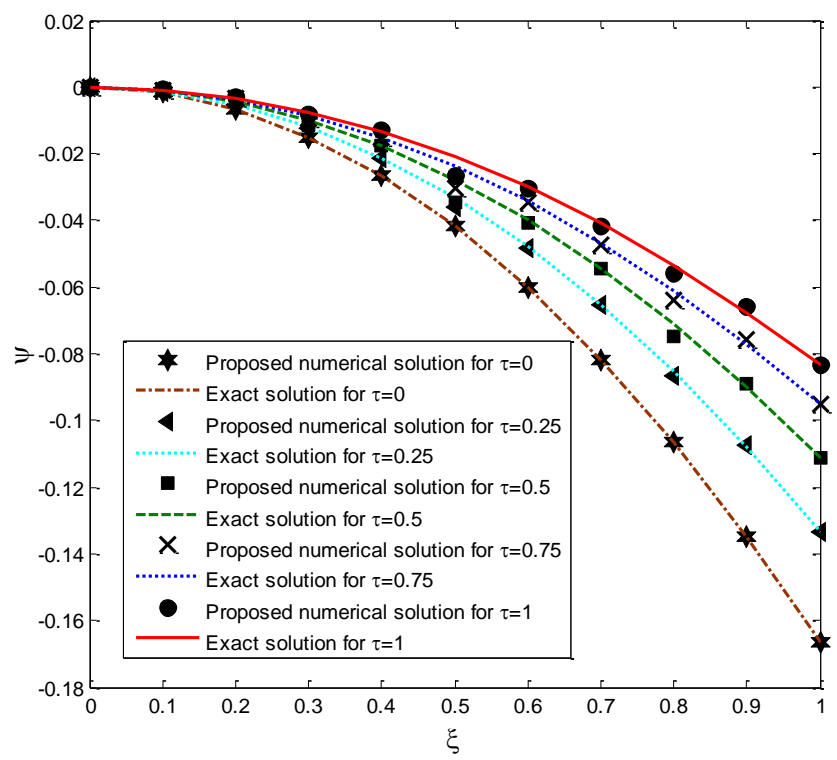

Fig. 29. Comparison of results obtained via the proposed method and exact solution for $h_{\tau}=$ 0.25 and $h_{\zeta}=0.1$ (Example 2). 


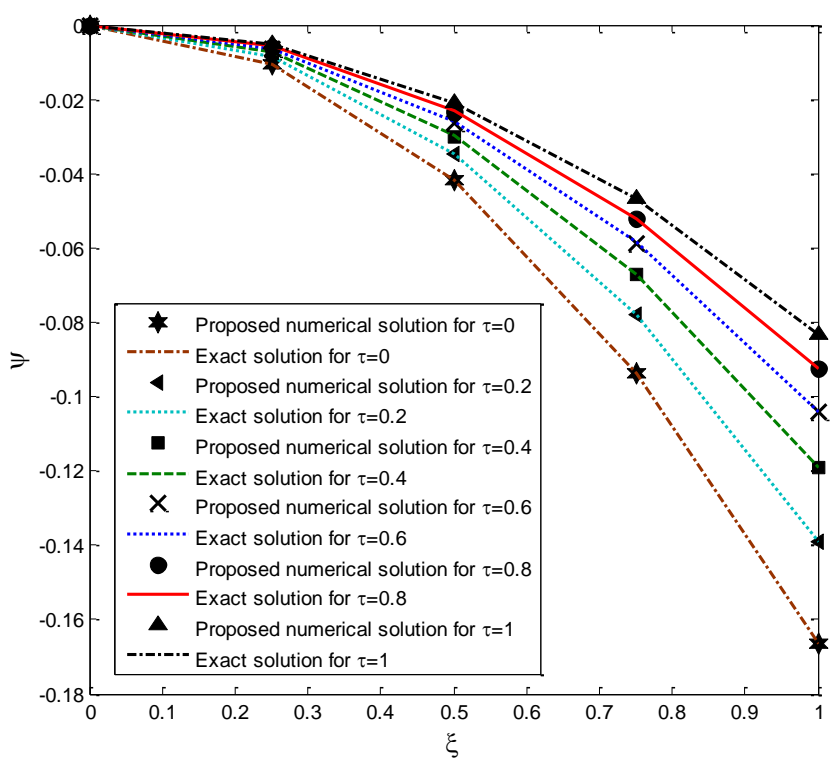

Fig. 30. Comparison of results obtained via the proposed method and exact solution for $h_{\tau}=$ 0.2 and $h_{\zeta}=0.25$ (Example 2).

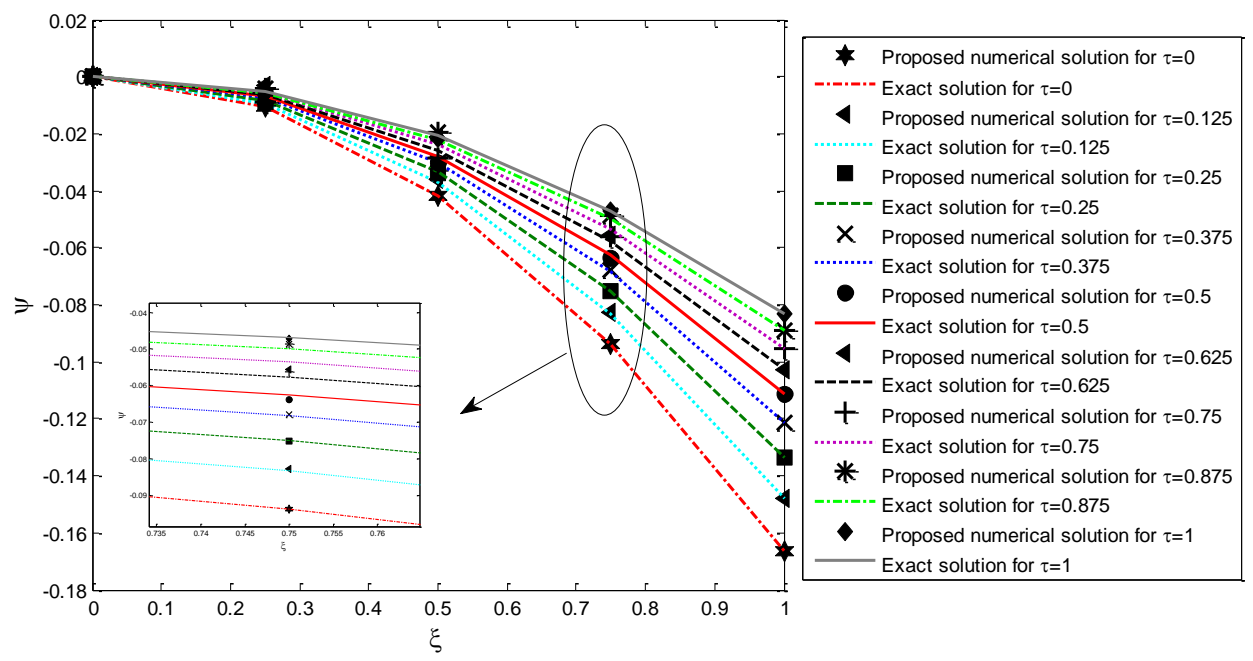

Fig. 31. Comparison of results obtained via the proposed method and exact solution for $h_{\tau}=$ 0.125 and $h_{\zeta}=0.25$ (Example 2).

\section{Conclusions}

In this study, a new combination of the optimization algorithms and the interpolation methods was described to analyze the nonlinear porous media differential equation. The moving least 
square was employed to produce the discretized function, and the penalty method was implemented to satisfy the initial and boundary conditions. Convergence trajectories and global optimum positions evidently articulated that the considered optimization algorithm, namely HEPSO, displays a higher performance and accuracy in comparison with the SPSO. Further, the solutions of the nonlinear porous media differential equation via the proposed strategy were compared with those of the analytical formulation for various number of regular nodes. These results illustrated that the introduced method is able to solve this class of nonlinear differential equations with an acceptable convergence speed and high accuracy without any restrictive assumptions.

\section{References}

Antoniouk A, Arnaudon M (2014). Variational principle for weighted porous media equation, Comptes Rendus Mathematique, Volume 352, Issue 1, January 2014, 31-34.

Campos J, Soler J (2016). Qualitative behavior and traveling waves for flux-saturated porous media equations arising in optimal mass transportation, Nonlinear Analysis, Volume 137, May 2016, 266-290.

Dareiotis K, Gerencsér M, Gess B (2019). Entropy solutions for stochastic porous media equations, Journal of Differential Equations, Volume 266, Issue 6, 5 March 2019, 37323763.

Doha EH, Hafez RM, Youssri YH (2019). Shifted Jacobi spectral-Galerkin method for solving hyperbolic partial differential equations, Computers \& Mathematics with Applications, Volume 78, Issue 3, 1 August 2019, 889-904.

Duan C, Liu C, Wang C, Yue X (2019). Numerical methods for porous medium equation by an energetic variational approach, Journal of Computational Physics, Volume 385, 15 May 2019, 13-32.

Duque JCM, Almeida RMP, Antontsev SN (2015). Application of the moving mesh method to the porous medium equation with variable exponent, Mathematics and Computers in Simulation, Volume 118, December 2015, 177-185.

Esmaeilbeigi M, Chatrabgoun O, M Shafa, (2019). Numerical solution of time-dependent stochastic partial differential equations using RBF partition of unity collocation method based on finite difference, Engineering Analysis with Boundary Elements, Volume 104, July 2019, 120-134.

Fakhar-Izadi F, Dehghan M (2018). Fully spectral collocation method for nonlinear parabolic partial integro-differential equations, Applied Numerical Mathematics, Volume 123, January 2018, 99-120.

Firoozjaee MA, Yousefi SA (2018). A numerical approach for fractional partial differential equations by using Ritz approximation, Applied Mathematics and Computation, Volume 338, 1 December 2018, 711-721.

Grillo G, Muratori M (2016). Smoothing effects for the porous medium equation on CartanHadamard manifolds, Nonlinear Analysis, Volume 131, January 2016, 346-362.

İzgi B, Çetin C (2018). Semi-implicit split-step numerical methods for a class of nonlinear stochastic differential equations with non-Lipschitz drift terms, Journal of Computational and Applied Mathematics, Volume 343, 1 December 2018, 62-79.

Jensen B, Jacobsen NG, Christensen ED (2014). Investigations on the porous media equations and resistance coefficients for coastal structures, Coastal Engineering, Volume 84, February 2014, 56-72.

Li C, Wang Z (2019). The local discontinuous Galerkin finite element methods for Caputo-type partial differential equations: Numerical analysis, Applied Numerical Mathematics, Volume 140, June 2019, 1-22. 
Mahmoodabadi MJ, Mahmoodabadi F, Atashafrooz M (2018). Development of the Meshless Local Petrov-Galerkin Method to Analyze Three-Dimensional Transient Incompressible Laminar Fluid Flow, Journal of the Serbian Society for Computational Mechanics 12 (2) 128-152.

Mahmoodabadi MJ, Mottaghi ZS, Bagheri A (2014). HEPSO: high exploration particle swarm optimization, Information Sciences, 273 101-111.

Pamuk S (2005). Solution of the porous media equation by Adomian's decomposition method, Phys. Lett. A 344 (2-4) 184-188.

Polyanin AD, Zaitsev VF (2004). Handbook of Nonlinear Partial Differential Equations, Chapman \& Hall/CRC Press, Boca Raton.

Röckner M, Wu W, Xie Y (2018). Stochastic porous media equation on general measure spaces with increasing Lipschitz nonlinearities, Stochastic Processes and their Applications, Volume 128, Issue 6, June 2018, 2131-2151.

Sibalija TV (2019). Particle swarm optimisation in designing parameters of manufacturing processes: A review (2008-2018), Applied Soft Computing, Volume 84, November 2019, Article 105743.

Yang Z, Zhang J, Zhao C (2019). Numerical blow-up analysis of linearly implicit Euler method for nonlinear parabolic integro-differential equations, Journal of Computational and Applied Mathematics, Volume 358, 1 October 2019, Pages 343-358.

Zeng F, Turner I, Burrage K, Wright SJ (2019). A discrete least squares collocation method for two-dimensional nonlinear time-dependent partial differential equations, Journal of Computational Physics, Volume 394, 1 October 2019, 177-199.

Zhou Y, Zhang C (2019). One-leg methods for nonlinear stiff fractional differential equations with Caputo derivatives, Applied Mathematics and Computation, Volume 348, 1 May 2019, pp. 594-608. 